\title{
Measurements of Gaseous Diffusion Coefficients by a Gas Chromatographic Technique
}

\author{
S. P. Wasik and K. E. McCulloh
}

Institute for Materials Research, National Bureau of Standards, Washington, D.C. 20234

(November 1, 1968)

\begin{abstract}
A method is presented for measuring gaseous diffusion coefficients using a gas chromatographic technique. Diffusion coefficients were measured for the systems: argon, krypton, oxygen, and nitrogen diffusing into helium at temperatures from 77 to $4.00 \mathrm{~K}$.

Key words: Argon; diffusion coefficient; gas chromatography; helium; krypton; nitrogen; oxygen.
\end{abstract}

\section{Introduction}

Accurate knowledge of molecular diffusion coefficients and their temperature dependence is important in obtaining fundamental information on forces between unlike molecules. Until recently most diffusion measurements were conducted over short temperature intervals because the conventional methods such as the Loschmidt or the Stefan, require the thermostating of large, static systems. Walker and Westenberg [1] ${ }^{1}$ have developed a method which involves measuring concentration profiles downstream of a point source of tracer gas in a uniform laminar jet of carrier gas. Diffusion coefficient for permanent gas pairs over a temperature interval 300 to $1100 \mathrm{~K}$ have been measured using this technique.

Determining diffusion coefficients using gas cromatographic techniques is relatively new. Taylor [2] derived an expression for the radial diffusion term for a liquid dispersion flowing in a tube. The same expression was derived by Giddings [3] and Golay [4] as a special case of a general theory of gas chromatography. Giddings and Seager [5] have measured diffusion coefficients using this method.

The method reported in this paper is also based on gas chromatography techniques. In brief, a trace amount of one gas was injected into a carrier gas stream of different composition flowing through a column at a constant flow rate. The concentration profile of the gas dispersion was determined at two points downstream from the point of sample injection by thermal conductivity detectors. The binary diffusion coefficients were calculated from the dimensions of the recorded peaks and time required for the peak

\footnotetext{
${ }^{1}$ Figure in brackets indicate the literature references at the end of this paper.
}

maximum to travel the distance between the two detectors.

Diffusion coefficients were measured for the systems: argon, krypton, oxygen and nitrogen diffusing into helium at temperatures from 77 to $400 \mathrm{~K}$.

\section{Theoretical}

\subsection{Fundamental Relations}

As has been shown by theoretical studies [2, 3, 4] when a nonuniform gaseous dispersion flows through a cylindrical tube, the effect of radial diffusion acting in conjunction with the parabolic velocity profile is accounted for by an effective longitudinal diffusion coefficient $\alpha^{2}$, given by

$$
\alpha^{2}=D+\frac{1}{48} \frac{v^{2} a^{2}}{D}
$$

where $v$ is the mean flow velocity, $a$ is the inside radius of the tube, and $D$ is the diffusion coefficient. If $c$ represents the mean concentration in a cross section at distance $x$ from the moving centroid of the dispersion ( $x$ is taken positive downstream from the centroid) then $c$ satisfies the one-dimensional diffusion equation

$$
\frac{\partial c}{\partial t}=\alpha^{2} \frac{\partial^{2} c}{\partial x^{2}}
$$

First let us assume an ideal system in which the experiment is initiated by instantaneous injection at time $t=0$ of $n$ moles of diffusing substance into the carrier gas stream at a fixed injection point, and 
further assume that the external parameters affecting the flow (e.g., temperature, pressure, and tube radius) are all constant. Equation (2) applies after a time which is long compared with $a^{2} / D$. Its solution appropriate to these initial conditions is given by

$$
c=\frac{n}{\pi a^{2}} \frac{1}{2 \alpha \sqrt{\pi t}} e^{-x^{2} / 4 \alpha^{2} t}
$$

Equation (3) defines a concentration distribution which is instantaneously Gaussian and spreading with time. In the actual system the assumption of constant flow parameters applies only to the diffusion column proper. However, the experimental system was designed such that the concentration distribution was always instantaneously Gaussian within the diffusion column. Equation (3) can be applied under these conditions if the zero of time is redefined. Instead of taking $t=0$ as the instant of sample injection, it must be so defined that eq (3) is consistent with an analysis of the peaks recorded from two or more separate detectors along the tube.

Equation (3) expresses concentration in terms of a frame of reference that moves with the mean velocity of the carrier gas stream. In order to express concentration as a function of time at a fixed detector, we must transform eq (3) by means of the relation

$$
x=v\left(t_{0}-t\right),
$$

where $t_{0}$ is that time at which the centroid of the dispersion arrives at the detector. When we make this substitution, the concentration at a fixed detector is given by

$$
c=\frac{n}{\pi a^{2}} \frac{1}{2 \alpha \sqrt{\pi t}} e^{-v^{2}\left(t_{0}-t\right)^{2} / 4 \alpha^{2} t}
$$

\subsection{Peak Analysis}

Assuming linear detector response, the height $h$ of a recorder trace of the signal at time $t$ is proportional to $c$ as expressed by eq (5). If $h_{0}$ is the height at time $t_{0}$, it follows from eq (5) that

$$
t \ln \frac{\sqrt{t_{0}} h_{0}}{\sqrt{t} h}=\frac{v^{2}}{4 \alpha^{2}}\left(t_{0}-t\right)^{2} .
$$

From this equation it is seen that a straight line with the slope $v^{2} / 4 \alpha^{2}$ should be obtained when

$$
t\left(\ln \frac{h_{0}}{h}+\frac{1}{2} \ln \frac{t_{0}}{t}\right)
$$

is plotted versus $\left(t_{0}-t\right)^{2}$. Such a plot is useful for demonstrating that the peak shape conforms to theory. However, for determining the diffusion coefficient an approximation based on eq (6) is easier to apply while providing sufficient accuracy.
This approximation can be derived by introducing the characteristic diffusion distance $s$ defined by

$$
s=\alpha \sqrt{t_{0}},
$$

the dimensionless variable $\eta$ defined by

$$
v\left(t-t_{0}\right)=2 \eta s,
$$

and the small dimensionless parameter $\epsilon$ defined by

$$
s=\epsilon v t_{0} .
$$

When eq (6) is rewritten in terms of $\epsilon$ and $\eta$, the result is

$$
(1+2 \epsilon \eta)\left[\ln \frac{h_{0}}{h}-\frac{1}{2} \ln (1+2 \epsilon \eta)\right]=\eta^{2}
$$

Since $\epsilon$ is small (typically, $\epsilon^{2}<10^{-3}$ ), eq (8) can be expanded in powers of $\epsilon$. Neglecting powers higher than the second,

$$
\left(1+\epsilon^{2}\right) \eta^{2}-\epsilon\left(2 \ln \frac{h_{0}}{h}-1\right) \eta-\ln \frac{h_{0}}{h} \cong 0 .
$$

This equation is quadratic in $\eta$. Let $\Delta \eta$ be the difference of its two roots. Expanding $\ln \frac{h_{0}}{h}$ as a power series in $\epsilon^{2}$, the solution of eq (8), correct through terms in $\epsilon^{2}$, can be written

$$
4 \ln \frac{h_{0}}{h}=(\Delta \eta)^{2}+\epsilon^{2}\left[2(\Delta \eta)^{2}-\frac{1}{4}(\Delta \eta)^{4}-1\right] .
$$

Taking typical values for $\epsilon$ and $\Delta \eta$ (typically, $\Delta \eta^{2}<10$ ), it can be readily seen that all but the first term on the right side of this equation can be neglected when accuracy to a few parts per thousand is adequate. If we let $\Delta t$ represent the width of the recorder trace at height $h$, we can make use of eqs (7a) and (7b) to write

$$
\Delta \eta=\frac{v}{\alpha \sqrt{t_{0}}} \frac{\Delta t}{2}
$$

Substituting in eq (9) and retaining only the first term on the right we obtain the approximation

$$
t_{0} \ln \frac{h_{0}}{h}=\frac{v^{2}}{4 \alpha^{2}}\left(\frac{\Delta t}{2}\right)^{2}
$$

If $t_{0} \ln \frac{h_{0}}{h}$ is plotted versus $\left(\frac{\Delta t}{2}\right)^{2}$, it is seen that a straight line with the slope $v^{2} / 4 \alpha^{2}$ should be obtained. This plot should superimpose accurately on that based on eq (6). It is obvious that eq (10) is easier to apply in determining $\alpha$.

Since $t_{0}$, the time of arrival of the peak at a detector, appears on the left in eq (10), it is still necessary to define the zero of time. The difference of the $t_{0}$ values 
at two detectors along the tube can be measured directly. Their ratio can be obtained by applying eq (10) to each of the detectors. Solving for the two $t_{0}$ from their difference and their ratio is equivalent to determining the effective zero of time.

Another method which, although tedious, yields $\alpha$ with good precision because random fluctuations in the recorder signal are smoothed by integration, is based on the ratio of peak area to peak height. The equation

$$
h=h_{0} \sqrt{\frac{t_{0}}{t}} e^{-v^{2}\left(t_{0}-t\right) 2 / 4 \alpha^{2} t}
$$

equivalent to eq (6), can be reduced to a standard integral form (7), by the substitution $y^{2}=v^{2} t / 4 \alpha^{2}$, with the result

$$
\left(\frac{\int_{0}^{\infty} h d t}{h_{0}}\right)^{2}=\frac{4 \alpha^{2} \pi t_{0}}{v^{2}} .
$$

\subsection{Experimental Procedure}

In figure 1 is shown a schematic drawing of the apparatus. A $150-\mathrm{ft}$ empty $1 / 4$-in O.D. copper column was divided into three equal segments; a thermostatic column, $\mathrm{C}_{1}$, the diffusion column, $\mathrm{C}_{2}$, and a third column, $\mathrm{C}_{3}$, which allowed the carrier gas to come to room themperature before its flow rate was measured by a soap-bubble flowmeter, F. The thermostating and diffusion columns were immersed in liquid nitrogen for the low temperature measurements and an oil thermostat, controlled to $\pm 0.02{ }^{\circ} \mathrm{C}$, for the other measurements. At the beginning and end of the diffusion column were thermal conductivity detectors, $\mathrm{D}_{1}$ and $\mathrm{D}_{2}$, located in line with the incoming gas flow. The detectors, shown in figure 2 , were constructed from $1 / 4$-in Tee connectors for tubing in which one arm was cut off to allow the sensing element, a Gow Mac type 9225 filament support (see footnote 2) to be soldered into position so that the sensing element was on the central axis of the diffusion column. The two

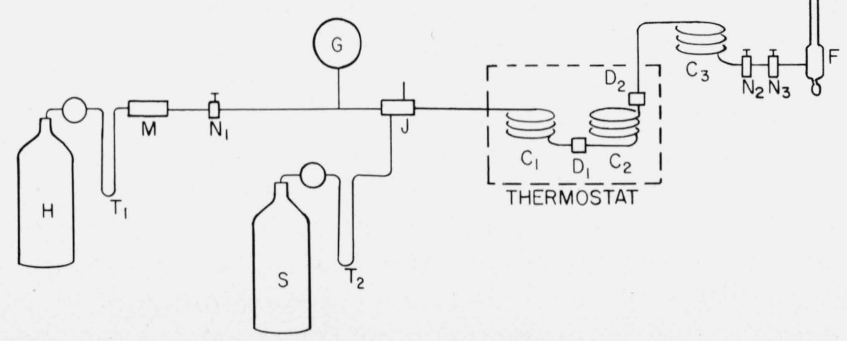

FigurE 1. Schematic diagram of flow system for measuring diffusion coefficients.

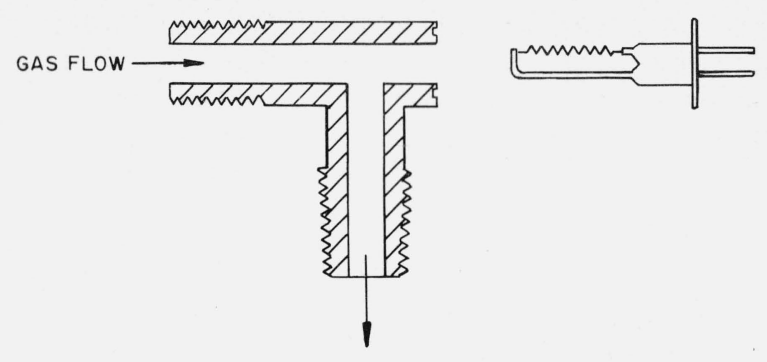

Figure 2. Detector.

detectors formed adjacent arms of a Wheatstone bridge circuit. The resultant voltages were plotted as a function of time using a $1 \mathrm{mV}$ full scale recorder.

The flow of helium carrier gas from a high pressure tank, H, was controlled by a Moore [6] ${ }^{2}$ constantdifferential type flow controller, M, a needle valve, $\mathrm{N}_{1}$, upstream from the diffusion column and two needle valves, $\mathrm{N}_{2}$ and $\mathrm{N}_{3}$, downstream from the diffusion column. An $0.1 \mathrm{ml}$ of sample gas from a high pressure tank, $\mathrm{S}$, was injected into the thermostating column by means of a F \& M [8] gas injection valve, J. Commercially available gases with a stated purity of better than 99 mole percent were dried by passing them through a molecular sieve column $\left(\mathrm{T}_{1}\right.$ and $\mathrm{T}_{2}$ ). The column pressure was read from a Wallace and Tiernan [9] absolute aneroid barometer, G.

The effective radius, $a$, was calculated at room temperature by equating the mass flow rate measured by the soap-bubble meter to the mass flow rate in the diffusion column, $\pi P_{c} a^{2} L / t$, where $P_{c}$ is the pressure of the carrier gas in atmospheres and $t$ is the time required for an air peak to travel the distance, $L$, between the two detectors. The value of a agreed within one part in two hundred for all the measurements taken.

For the high and low temperature measurements the mass flow rate in the diffusion column was used to determine the temperature of the carrier gas, the column's dimensions being corrected for thermal expansion. If the carrier gas was not at the temperature of the thermostat the flow rate was reduced until it was.

A preliminary diffusion measurement was made for each system to determine peak heights. The attenuation was then adjusted by means of a 10-turn, 10,000 $\Omega$ Heli-pot so that for all the following measurements the peak heights would be the maximum height allowed by the recorder chart paper. Duplicate runs were made by injecting successive amounts of sample gas into the carrier gas stream, the injections being at a time interval such that no overlapping of peaks occurred. At liquid nitrogen temperature, it was possible for as many as four peaks to be in the diffusion column simultaneously. This enables four velocity measurements to be made during the course of a run. The time

${ }^{2}$ Certain commercial materials and equipment are identified in this paper in order to specify adequately the experimental procedure. In no case does such identification imply recommendation or endorsement by the National Bureau of Standards, nor does it imply that the material or equipment identified is necessarily the best available for the purpose. 
between peaks was checked with a stop watch. The heights and widths of the peaks were measured to a precision of one part in two hundred fifty with an engineer's scale.

The quantity $\alpha^{2}$ was calculated from the expression

$$
\alpha^{2}=\left(\frac{1}{S_{2}}-\frac{1}{S_{1}}\right) \frac{v^{2}}{16\left(t_{02}-t_{01}\right)}
$$

where $S_{2}$ and $S_{1}$ are the slopes of the $\ln H_{0} / h-(\Delta t)^{2}$ plots and $\left(t_{02}-t_{01}\right)$ is the time required for the peak maximum to travel the distance between detectors.

The quantity $\alpha^{2}$ was also calculated from

$$
\left(\frac{A_{2}}{h_{02}}\right)^{2}-\left(\frac{A_{1}}{h_{01}}\right)^{2}=\frac{4 \alpha^{2} \pi\left(t_{02}-t_{01}\right)}{v^{2}}
$$

where $A_{2}$ and $A_{1}$ are the areas under the second and first peak respectively and $v$ the velocity of the carrier gas in the diffusion column. Equation (14) which follows from eq (11), gave the same values for $\alpha^{2}$ as eq (13).

\section{Results and Discussion}

In figure 3 are shown plots of eqs (6) and (10) for one experiment on the diffusion of nitrogen in helium at 30 psia and $25^{\circ}$. The filled squares represent values calculated from eq $(10)$ for the first peak with $t_{01}$ equal to $23.59 \mathrm{~min}$ and the open squares are values obtained from the second peak with $t_{02}$ equal to $48.81 \mathrm{~min}$. The open circles represent values calculated from eq (6) from the leading half of the first peak while the filled circles are those of the leading half of the second peak. The half-filled circles right represent values calculated from eq (6) from the trailing half of the first peak while the half-filled circles left are the corresponding values for the second peak. All experimental points fit the theoretical line within a precision of 0.5 percent.

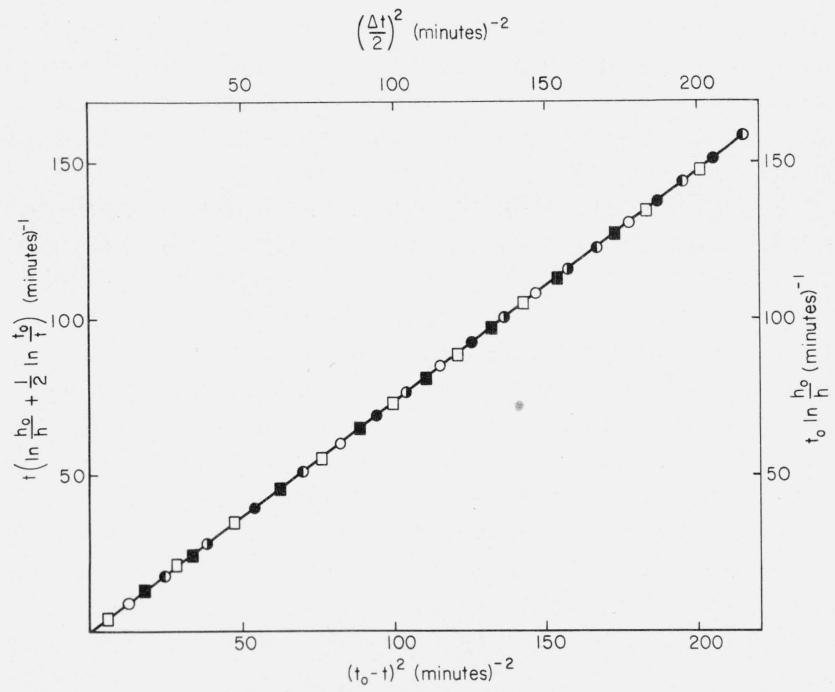

Figure 3. Plot of eqs (6) and (10) for the diffusion of nitrogen in helium at 30 psia and $25^{\circ} \mathrm{C}$.
There are no indications of skewing or tailing of either peak.

Values for the diffusion coefficients at different temperatures are given in table 1 . The pressure of the carrier gas in the diffusion column varied from 1.1 to $1.6 \mathrm{~atm}$ for these determinations. These values have been corrected to standard atmospheric pressure. Each diffusion coefficient was obtained as an average of eight separate determinations. The values of 0.732 and $0.688 \mathrm{~cm}^{2} / \mathrm{s}$ for the diffusion of argon in helium and nitrogen in helium at $25{ }^{\circ} \mathrm{C}$ respectively compare favorably with values of 0.725 and $0.688 \mathrm{~cm}^{2} / \mathrm{s}$ obtained by Walker and Westenberg [1] for the same gas pairs.

TABLE 1. Effect of Temperature on the Diffusion Coefficient

\begin{tabular}{c|c|c}
\hline \hline & Temp. K & $D\left(\mathrm{~cm}^{2} / \mathrm{s}\right)$ \\
\hline Gas pair & & \\
$\mathrm{He}-\mathrm{A}$ & 77.2 & $0.0710 \pm 0.0009 *$ \\
$\mathrm{He}-\mathrm{A}$ & 298 & $.7335 \pm .004$ \\
$\mathrm{He}-\mathrm{A}$ & 334 & $.8890 \pm .006$ \\
$\mathrm{He}-\mathrm{A}$ & 357 & $.9917 \pm .006$ \\
$\mathrm{He}-\mathrm{N}_{2}$ & 77.2 & $.0725 \pm .001$ \\
$\mathrm{He}-\mathrm{N}_{2}$ & 296 & $.678 \pm .005$ \\
$\mathrm{He}-\mathrm{N}_{2}$ & 321 & $.8171 \pm .005$ \\
$\mathrm{He}-\mathrm{N}_{2}$ & 348 & $.9251 \pm .007$ \\
$\mathrm{He}-\mathrm{N}_{2}$ & 370 & $1.030 \pm .006$ \\
$\mathrm{He}-\mathrm{Kr}$ & 298 & $.6491 \pm .004$ \\
$\mathrm{He}-\mathrm{Kr}$ & 322 & $.7372 \pm .004$ \\
$\mathrm{He}-\mathrm{Kr}$ & 341 & $.813 \pm .005$ \\
$\mathrm{He}-\mathrm{Kr}$ & 366 & $.904 \pm .006$ \\
$\mathrm{He}-\mathrm{O}_{2}$ & 298 & $.7361 \pm .005$ \\
$\mathrm{He}-\mathrm{O}_{2}$ & 320 & $.8472 \pm .006$ \\
$\mathrm{He}-\mathrm{O}_{2}$ & 365 & $1.041 \pm .008$ \\
\hline
\end{tabular}

* $90 \%$ confidence limits for the mean

A least square calculation for the expression

$$
\log \frac{D_{298}}{D_{T}}=m \log \frac{298}{T}
$$

where $D_{298}$ is the diffusion coefficient at $298 \mathrm{~K}, D_{T}$ is the diffusion coefficient at temperature, $T$, and $m$ is a constant. The following values for $m$ were obtained with indicated precision being for a 90 percent confidence limit: $\mathrm{He}-\mathrm{N}_{2}, 1.69 \pm 0.02 ; \mathrm{He}-\mathrm{O}_{2}, 1.67 \pm 0.06$; $\mathrm{He}-\mathrm{Kr}, 1.71 \pm 0.06$ and He-Ar, $1.72 \pm 0.006$. These values may be compared with values obtained by Seager, Geerston, and Giddings [5]: $\mathrm{He}-\mathrm{N}_{2}, 1.75 \pm 0.12$; $\mathrm{He}-\mathrm{O}_{2}, 1.70 \pm 0.08$ and $\mathrm{He}-\mathrm{Ar}, 1.73 \pm 0.07$. The two sets agree within the given experimental errors. In figure 4 is shown the log diffusion coefficient-log temperature plot for argon diffusing into helium.

There are several advantages to this method.

(a) The use of two detectors instead of one eliminates the need for a zero time correction, since it is not necessary to know the shape of the peak at the point of sample injection. The only requirements are that the concentration profile be Gaussian when the peak reaches the first detector, that the thermal conductivity detectors do not distrub the concentration profile, and that the carrier gas be at thermostat temperature since the sample injection value is at room temperature.

(b) Diffusion coefficients over a wide range of temperature may be measured since the apparatus may be used in a liquid nitrogen bath or in an oven without any change in design. 


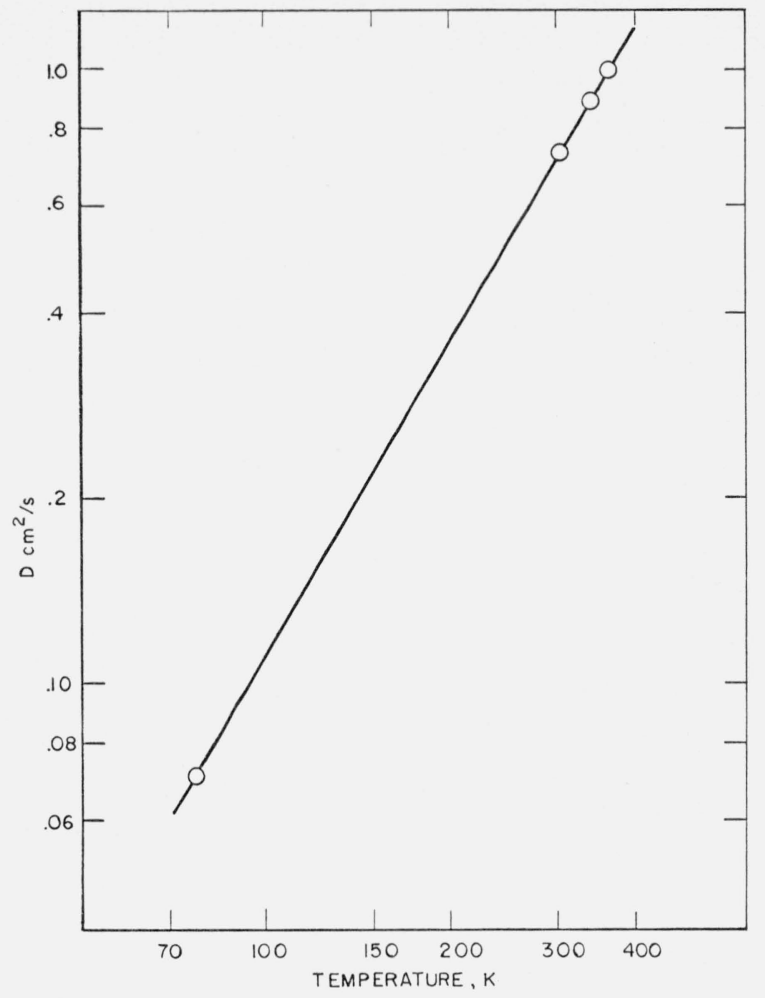

Figure 4. Plot of log diffusion coefficient-log temperature for argon diffusing into helium.

(c) Time measurements may be made accurately. Since the velocity of the carrier gas appears as a square term in the diffusion equation it is important to be able to measure this quantity accurately. This can be done by measuring the time required for the peak maxima to travel the measured distance between the two detectors. By injecting more than one peak into the diffusion column it is possible to determine whether the velocity of the carrier gas has changed during a measurement.

(d) Deviations of experiment from the assumed model may be determined. Equation (6) provides a means for analyzing the peak for any disturbance due to gas absorption on the walls of the diffusing column or for any disturbance to the concentration profile due to the gas injection and detection systems.

It is conluded that this is a simple, rapid, widely applicable method for the determination of gaseous diffusion coefficients.

\section{References}

[1] Walker, R. E., and Westenberg, H. A., J. Chem. Phys. 31, 519 (1959).

[2] Taylor, G. I., Proc. Roy. Soc. A223, 446 (1954).

[3] Giddings, J. C., J. Chem. Phys. 26, 169 (1957).

[4] Golay, M., Gas Chromatography (butterworths, London, 1958), p. 35, (editor, D. H. Desty).

[5] Seager, S. L., Geertson, L. R., and Giddings, J. C., J. Chem. Eng. Data 8, 168 (1963).

[6] Moore Products Co., Philadelphia, Pa.

[7] Gow-Mac Instrument Co., Madison, New Jersey.

[8] F \& M Scientific Corp., Avondale, Pa.

[9] Wallace and Tiernan Inc., Belleville, New Jersey.

(Paper 73A2-546) 